\title{
Modeling and Experiment of the Current Limiting Performance of a Resistive Superconducting Fault Current Limiter in the Experimental System
}

\author{
Fei Liang ${ }^{1}$ - Weijia Yuan ${ }^{1}$ - Carlos A. Baldan ${ }^{2,3} \cdot$ Min Zhang1 • Jérika S. Lamas ${ }^{4}$
}

Received: 18 March 2015 / Accepted: 6 May 2015 / Published online: 22 May 2015

(c) Springer Science+Business Media New York 2015

\begin{abstract}
In this paper, a 220-V resistive superconducting fault current limiter (SFCL) prototype is built and tested under different prospective fault currents, which vary from 0.8 to $7.4 \mathrm{kA}$. A 2D superconductor model is integrated into an experimental circuit model in COMSOL to simulate the performance of the SFCL prototype in the experimental system in fault tests. In the simulation, a new $E$ - $J$ relationship is proposed to enhance the convergence of calculation. Comparison between simulation results and experimental results shows that the proposed model performs well in simulating current limiting performance of SFCL in experimental system in case of fault.
\end{abstract}

Keywords Finite element method - YBCO .

Superconducting fault current limiter - SFCL .

COMSOL $\cdot \mathrm{H}$ formulation

Weijia Yuan

W.Yuan@bath.ac.uk

1 Department of Electronic and Electrical Engineering,

University of Bath, Claverton Down,

Bath, BA2 7AY, UK

2 School of Engineering of Lorena, University of Sao Paulo, EEL-USP, Lorena, SP, Brazil

3 Department of Electrical Engineering, State University of Sao Paulo, UNESP, Guaratinguetá, SP, Brazil

4 Faculty of Sciences, Analytical and Interfacial Chemistry (CHANI), Université Libre de Bruxelles, Brussels, Belgium

\section{Introduction}

With the increase of interconnection of transmission lines and new generation sources, the problem of excessive fault currents in power grids is becoming more and more severe today [1]. Resistive type SFCLs, which are based on the transition of superconductor from superconducting state to normal state, is one of the most promising superconducting fault current limiters (SFCLs) that can solve the problems.

In normal operation, the resistance of SFCL is almost zero. However, when short circuit occurs, SFCL acts like a resistor and cooperates with circuit breakers to clear the fault. The current limiting performance of SFCL determines whether the fault current can be limited to a value that circuit breaker can cut off. Therefore, the simulation of the current limiting performance of SFCL is very important.

So far, many finite element models have been proposed to study the quench process of high-temperature superconductors. F. Roy [2, 3] proposed a 2D electro-magneto-thermal model, which was calculated with $H$ formulation, to simulate the quench performance of SC tape. The increase of the thickness of each layer of superconductor, aiming to speed up calculation, might result in inaccuracy of calculation result. Similarly, a 2D axisymmetric model using $H$ formulation was proposed to calculate the quench propagation of superconducting coils in the case of heat pulses $[4,5]$. In the model, a simplified superconductor model was used by deleting the $\mathrm{Ag}$ and buffer layers to enable fast calculation. Meanwhile, several 3D models have also been presented to investigate the quench process. J Duron [6]. proposed a 3D FEM model to study the quench behavior of the superconductor with a method based on fast-forward scheme, where the electromagnetic model and thermal model are calculated alternately. In the simulation, the geometric aspect ratio was decreased by increasing the thickness of YBCO and 
Ag. Wan Kan Chan [7-9] proposed a 3D micrometer-scale model, which coupled 2D model and 3D model to simulating the quench propagation in both $\mathrm{SC}$ tapes and coils under DC conditions. Besides, a hybrid model, which combines the finite element method and a current repartition Matlab function, has also been used to simulate the quench propagation of superconductor [10]. However, the model did not take into account the influence of magnetic field to current redistribution during simulation.

As shown above, most finite element models concerning quench mainly focus on microscopic characteristics of superconductor, such as the quench performance and quench propagation of superconductor. However, neither of them has been used to simulate the current limiting performance of SFCL as a device in power system, which can be realized by coupling a SFCL model and a power system model. Simulating the performance of SFCL in its operating environment enables researchers and engineers to better understand the requirement of its operation environment to SFCL. What is more, it can also be used to evaluate the effect of SFCL on power grids in case of fault, which is more important and useful to power network operators [11]. Therefore, a finite element model is integrated into an experimental circuit model in COMSOL to simulate the current limiting performance of a resistive SFCL prototype in experimental system under different fault currents in this paper. The goal of this paper is to demonstrate the possibility of the model proposed in simulating the current limiting performance of large scale of SFCL in power system.

The 220-V SFCL prototype is consisted of 16 elements connected in series, each element containing four YBCO tapes and a shunt in parallel [12]. To ensure the accuracy of calculation results, the real dimensions of superconducting tapes are used in the simulation. To enhance the convergence of calculation, a new $E-J$ relationship is proposed to describe the electrical characteristic of YBCO in the simulation.

In Section 2, the SFCL prototype and the corresponding 2D superconductor model are introduced. In Section 3, the experimental circuit and the simulation model are presented. In Section 4, the comparison of simulation results and experimental results is provided. In Section 5, some problems in calculation are discussed. Conclusions are presented in Section 6.

\section{SFCL Prototype and Superconductor Model}

\subsection{Introduction of SFCL Prototype}

The SFCL prototype, which is shown in Fig. 1, consisted of 16 elements connected in series. Each element contains four superconducting tapes and an external $180-\mathrm{m} \Omega$ shunt resistor, which are connected in parallel, as illustrated in Fig. 2

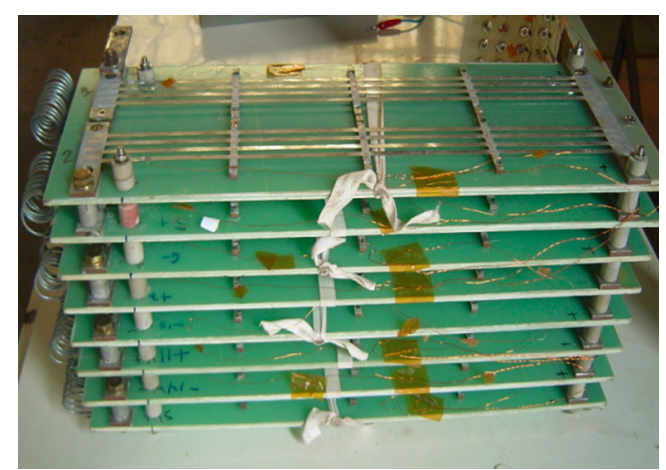

Fig. 1 SFCL prototype

[12]. The tape used in the SFCL prototype is American Superconductor's $344 \mathrm{~S}$ tape, with critical current $72 \pm 2 \mathrm{~A}$, width $4.4 \mathrm{~mm}$, and thickness $0.15 \mathrm{~mm}$. The parameters of the tape is shown in Table 1 [13]. The effective length of each tape is $0.4 \mathrm{~m}$; therefore, the total length of tape used in the SFCL prototype is $25.6 \mathrm{~m}$.

To simulate the SFCL prototype, a 2D finite element model is built in COMSOL, where the cross-section of the four superconducting tapes is used to express the superconducting tapes of each element of SFCL prototype, as shown in Fig. 3. Considering that the shunt in each element of the SFCL prototype is difficult to simulate in the 2D superconductor model, it is simulated in the experimental circuit model, which will be introduced in Section 3.2. For the simulation of $344 \mathrm{~S}$ tape, the real SC tape structure and geometry are used, as shown in Fig. 4. Each tape consisted of seven layers: two SUS 316L layers, two solder layers, a $\mathrm{Ni}-5$ at \%W layer, a YBCO layer, and a silver layer, with the geometry of each tape shown in Table 1.

The electrical and thermal parameters of substrate, HTS, protective, solder, and stabilizer used in the simulation are listed in Table 2 [13], and some detailed temperaturedependent variables are provided in the Appendix.

\subsection{Superconductor Model}

Considering that the quench process is not only an electromagnetic process, but also a thermal one, the

Tape

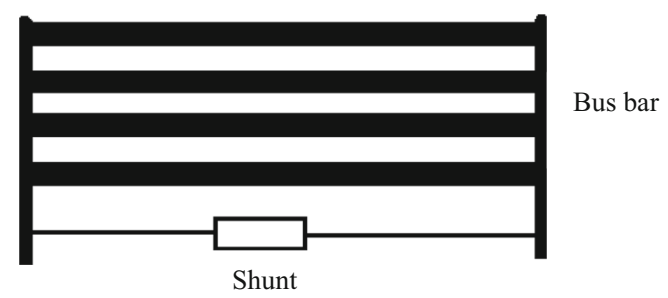

Fig. 2 Schematic diagram of each element of SFCL prototype 
Table 1 Parameter of AMSC 344S tape [13]

\begin{tabular}{ll}
\hline Substrate & Ni-5at $\% \mathrm{~W}(75 \mu \mathrm{m})$ \\
HTS & YBCO $(1 \mu \mathrm{m})$ \\
Protective & $\mathrm{Ag}(4 \mu \mathrm{m})$ \\
Solder & Mixture $(\mathrm{Sn}, \mathrm{Pb}, \mathrm{Ag})(3+3 \mu \mathrm{m})$ \\
Stabilizer & Stainless steel 316L (SUS 316L) $(25+25 \mu \mathrm{m})$ \\
\hline
\end{tabular}

superconducting tapes are simulated by coupling an electromagnetic model and a thermal model. For the electromagnetic model, the four parallel tapes are calculated with only one partial differential equations (PDE) in COMSOL. For the thermal model, each tape is calculated with a separate PDE, considering that there is no direct thermal contact between the four parallel tapes.

\subsubsection{Equations of Electromagnetic Model}

$H$ formulation is used to solve the electromagnetic property of the 2D model shown in Section 2.1 [26]. In the model shown in Fig. 3, the superconductor length is assumed to be infinite in the $Z$ direction. Current flows only in the $Z$ direction and magnetic field exists in the $X Y$ plane only. Equation (1) is used to express the $E-J$ relationship of superconductor, where $\rho$ is a variable.

$E=\rho * J$

$E$ and $J$ mean the electric field and the current density of superconductor, respectively. For different layers of superconducting tape, $\rho$ means the resistivity of corresponding layer.

By combining Faraday's law, Ampere's law, and (1), a PDE can be obtained [26]

$\mu_{0} \frac{\partial \mu_{\mathrm{r}}}{\partial t} H+\mu_{0} \mu_{\mathrm{r}} \frac{\partial H}{\partial t}+\nabla \times(\rho \nabla \times H)=0$ where $\mu_{0}$ is the vacuum permeability, $\mu_{\mathrm{r}}$ means the relative permeability, and $H$ means the magnetic field.

According to $E-J$ power law, the electrical field in superconductor increases exponentially with current density, which is inconsistent with the reality that the resistivity of superconductor will reach the normal resistivity eventually. Therefore, the $E-J$ power law cannot be used to simulate the $E-J$ relationship when the current of superconductor is much higher than its critical current. Up to now, different equations have been proposed to describe the $E-J$ relationship of superconductor during quench. Joseph Duron $[6,27]$ proposed a black-box model, which took superconductor as the parallel connection of a nonlinear resistor, representing the superconducting material, and a normal resistor, meaning the metallic sheath. François Roy [3] described the electrical property of superconductor with a nonlinear resistivity, which varied with current density and temperature. W. Paul [28] defined the $E-J$ relationships of three operating states of superconductor-superconducting state, flux flow state, and normal conductor with $E \sim j^{\alpha}$, $E \sim j^{\beta}$, and $E=\rho j$, respectively. The $E-J$ power law can be modified by setting an upper limit to the resistivity of superconductor, as shown in (3), where the resistivity of superconductor that is larger than $\rho_{\text {norm }}$ is regarded as $\rho_{\text {norm }}$. $\rho_{\text {norm }}$ is the normal resistivity of superconductor, which is set to be a constant in the simulation. $J_{\mathrm{c}}$ is the critical current density of superconductor, and $E_{0}$ is the electric field in superconductor when the current density equals $J_{\mathrm{c}}$, which is taken as $1 \mu \mathrm{V} / \mathrm{cm}$.

$\rho_{\mathrm{sc} 1}=\min \left(\rho_{\text {norm }}, \frac{E_{0}}{J_{\mathrm{c}}}\left(\frac{J_{\mathrm{z}}}{J_{\mathrm{c}}}\right)^{n_{\text {_value }}-1}\right)$

Although (3) can be used to simulate the $E$ - $J$ relationship in the finite element simulation, there is a singularity on the $E-J$ curve, which easily leads to non-convergence in calculation. In order to improve the convergence of computation, a new $E-J$ relationship with a smooth transition from superconducting state to normal state is proposed in

Table 2 Electrical and thermal parameters

\begin{tabular}{lllll}
\hline Layer & Density & Resistivity & Heat capacity & Thermal conductivity \\
\cline { 2 - 5 } & $\mathrm{kg} / \mathrm{m}^{3}$ & $\Omega \mathrm{m}$ & $\mathrm{J} /(\mathrm{kg} \mathrm{K})$ & $\mathrm{W} /(\mathrm{m} \mathrm{K})$ \\
SUS316L & 8000 & Figure $18[14]$ & Figure $19[15]$ & Figure 19 [16] \\
Solder & $8410[17]$ & $1.40 \mathrm{e}-7[17]$ & $167[18]$ & $50[17]$ \\
Ni-5at $\% \mathrm{~W}$ & $10,400[19]$ & $2.68 \mathrm{e}-7[20]$ & 440 & Figure 20 [21] \\
YBCO & 5900 & $(5)$ & Figure 21 [22] & $17[4]$ \\
Ag & 10500 & $1.59 \mathrm{e}-8 \times(1+0.0038 \times(\mathrm{u} 3-293))[23]$ & 235 & Figure 22 [24, 25] \\
\hline
\end{tabular}

Due to the fact that the heat capacity and thermal conductivity of SUS $316 \mathrm{~L}$ are not found, the corresponding parameters of SUS 316 are used instead 
this paper. The new relationship is based on an exponential function, which is shown in (4) and (5), and two fixed points of the modified $E-J$ power law (3): $\left(J_{\mathrm{c}}, E_{0} / J_{\mathrm{c}}\right)$ and $\left(\alpha^{*} J_{\mathrm{c}}, \rho_{\text {norm }}\right) . \alpha$ is a coefficient that determines the trend of superconductor resistivity with current density. The first is the point where the current density equals critical current density, while the second one corresponds the point where the resistivity of superconductor equals normal resistivity.

The equations of this new $E-J$ relationship are shown in (4-7).

$E_{z}=\rho_{s c 2} * J_{z}$

$\rho_{\mathrm{sc} 2}=\left\{\begin{array}{cl}\rho_{\text {norm }} * e^{-\left(\frac{\left|J_{\mathrm{Z}}\right|-\beta * J_{c x}}{\gamma}\right)^{2}}, & 0 \leq J_{\mathrm{z}} \leq \beta * J_{\mathrm{cx}} \\ \rho_{\text {norm }} & , \beta * J_{\mathrm{cx}} \leq J_{\mathrm{z}}\end{array}\right.$

By taking the two points into (5), the two coefficients $\beta$ and $\gamma$ can be solved.

$\beta=\alpha$

$\gamma=\frac{(\beta-1) \times J_{\mathrm{cx}}}{\sqrt{-\ln \left(\frac{E_{0}}{J_{\mathrm{cx}} \times \rho_{\mathrm{norm}}}\right)}}$

where $\rho_{\mathrm{sc}}$ is the resistivity of superconducting layer, and $\beta$ and $\gamma$ are the coefficients that are used to define the new $E-J$ relationship of superconductor. $J_{\mathrm{cx}}$ is the critical current density of superconductor, which is usually taken as a function of temperature and magnetic field. For the definition of $J_{\mathrm{cx}}$, only the influence of temperatures on the critical current is considered due to the fact that it is much greater than the influence of magnetic field in the superconductor as in the arrangement shown in this paper. Therefore, $J_{\mathrm{cx}}$ is expressed by (8) [3].

$J_{\mathrm{cx}}=J_{\mathrm{c} 0} *\left(\frac{T_{\mathrm{c}}-T}{T_{\mathrm{c}}-T_{0}}\right)^{1.5}$

where $J_{\mathrm{c} 0}$ means the critical current density of superconductor when the temperature of superconductor equals $T_{0}$.

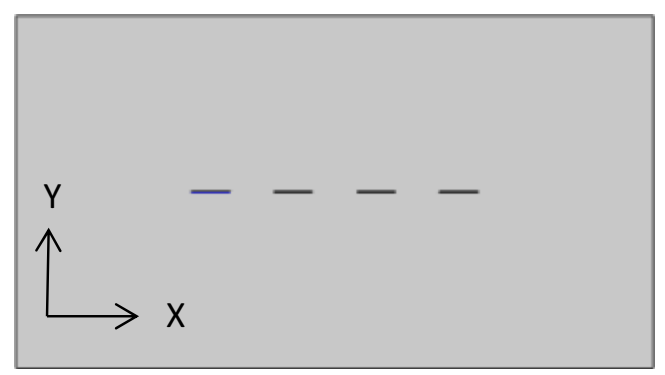

Fig. 3 2D model of four parallel $344 \mathrm{~S}$ tape

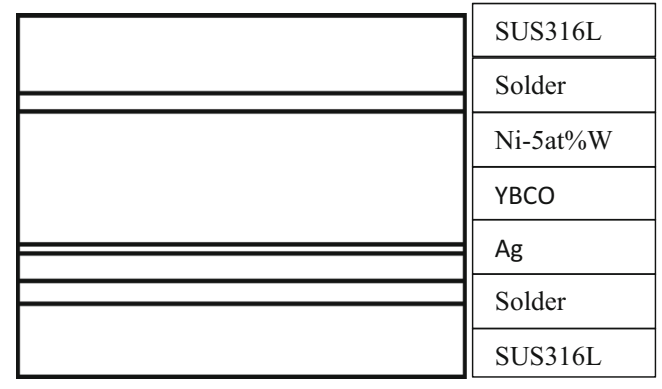

Fig. 4 Schematic diagram of each 344 S tape (not to scale)

$T_{0}$ means the temperature of liquid nitrogen, which is considered as $77 \mathrm{~K}$ here. $T_{\mathrm{c}}$ means the critical temperature of superconductor, which is regarded as $90 \mathrm{~K}$ here.

As shown in (4-7), the only variable of the new E-J relationship is $\alpha$.

The physical basis of the new $E$ - $J$ relationship is that the quench process is basically a process of heat dissipation. When the current is smaller than the critical current, the electric field on the superconductor is very small, which can be derived from the point of $\left(J_{\mathrm{c}}, E_{0} / J_{\mathrm{c}}\right)$ and (4) and (5); therefore, the heat dissipation can be neglected. However, when the current increases above the critical current, the electric field will increase exponentially, which leads to heat generation. Nevertheless, the electric field will not always increase exponentially, because finally the normal resistivity of superconductor will be reached, when the electrical field increases linearly with current density. In this paper, to express the $E-J$ relationship of superconductor more accurately $\alpha$ of the new $E-J$ relationship is chosen to make the $E-J$ relationship as close as the modified $E-J$ power law (3). The coefficient $\alpha$ of the new $E-J$ relationship is taken as

$\alpha=1.15 *\left(\frac{\rho_{\text {norm }} * J_{\mathrm{cx}}}{E_{0}}\right)^{\frac{1}{n_{\text {value }-1}}}$

where $\mathrm{n}$ value is taken as 31 , which is also used in the simulation.

Then, to better illustrate the new $E-J$ relationship, $\rho-J$ curves of the new $E-J$ relationship is compared with that of the modified $E-J$ power law and Duron's equation, which is shown in (10) and (11) [27].

$\rho_{\mathrm{sc}}=\frac{E_{0}}{J_{\mathrm{cx}}}\left(\frac{J_{\mathrm{z}}}{J_{\mathrm{cx}}}\right)^{n_{-} \text {value }-1}$ 
Fig. 5 Comparison of different $E J$ equations. a $J_{\mathrm{c}}(T)=J_{\mathrm{c} 0} \cdot \mathbf{b}$ $J_{\mathrm{c}}(T)=J_{\mathrm{c} 0} / 100 . \mathbf{c}$

$\mathrm{J}_{\mathrm{c}}(T)=J_{\mathrm{c} 0} / 10^{4} . \mathbf{d}$

$J_{\mathrm{c}}(T)=J_{\mathrm{c} 0} / 10^{7}$

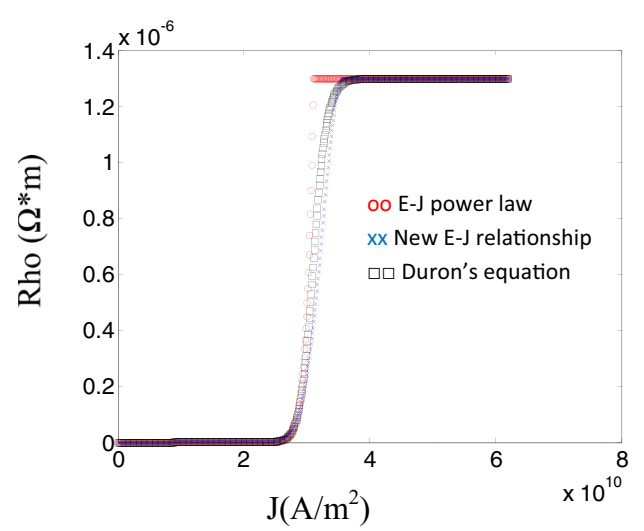

(a) $\mathrm{J}_{\mathrm{c}}(\mathrm{T})=\mathrm{J}_{\mathrm{c} 0}$

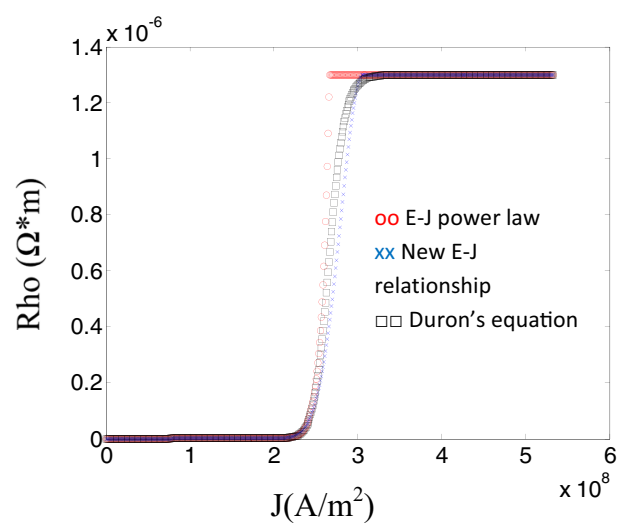

(b) $\left.\mathrm{J}_{\mathrm{c}} \mathrm{T}\right)=\mathrm{J}_{\mathrm{c} 0} / 100$

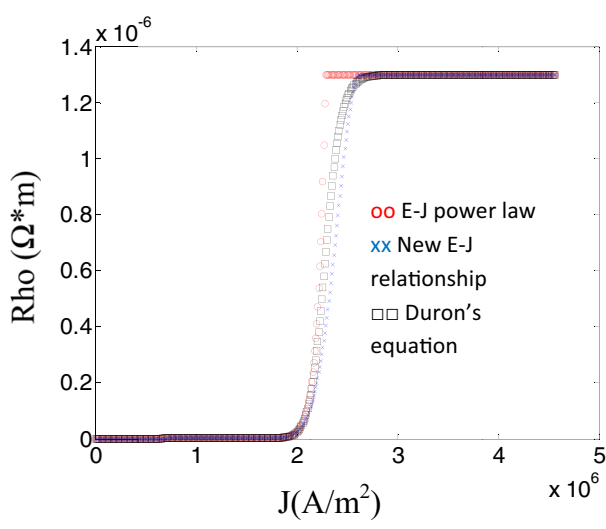

(c) $\mathrm{J}_{\mathrm{c}}(\mathrm{T})=\mathrm{J}_{\mathrm{c} 0} / 10^{4}$

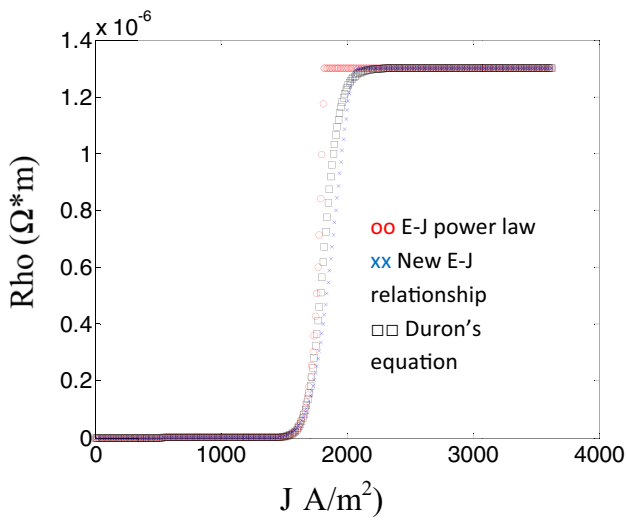

(d) $\mathrm{J}_{\mathrm{c}}(\mathrm{T})=\mathrm{J}_{\mathrm{c} 0} / 10^{7}$, $\rho_{\mathrm{sc} 3}=\frac{\rho_{\mathrm{sc}} * \rho_{\mathrm{norm}}}{\rho_{\mathrm{sc}}+\rho_{\mathrm{norm}}}$

As shown in Fig. 5, there is only little difference between three curves when the current is lower and slightly higher than the critical current $J_{\mathrm{cx}}$. Then with the increase of the current density, the slope of the new $E-J$ relationship curve and Duron's equation curve become smaller than that of the $E-J$ power law curve. Considering the fact that the superconducting layer is sandwiched between non-superconducting layers, most current shunts to the non-superconducting layer when the resistivity of superconductor increases to a high value, which leads to little heat dissipation at the superconductor layer. Therefore, the reduced slope of the new $E-J$ curve has little influence to the quench simulation. What is more, the slope reduction of the new $E-J$ curve can enhance the convergence of calculation, which is analyzed in detail in Section 5.1.

\subsubsection{Thermal Model}

For calculation of the thermal property of superconducting tapes during quench, a thermal equilibrium equation is built, as shown in (12),

$Q=\rho_{\mathrm{m}} C_{\mathrm{p}} \frac{\partial T}{\partial t}-\nabla(-k \nabla T)=E_{\mathrm{z}} J_{\mathrm{z}}$

where $Q$ means the power density in superconductor, $\rho_{\mathrm{m}}$ means density, $C_{\mathrm{p}}$ means the heat capacity, $k$ means the thermal conductivity.

What is more, in order to simulate the physical process of heat transfer from the superconducting tape to the liquid nitrogen, where the SFCL is placed, a heat transfer equation (13) is applied to the surface of superconductor in calculation [3].

$\hat{n} \cdot\left(k_{i} \nabla T\right)=h\left(T_{S}-T_{0}\right)$ 


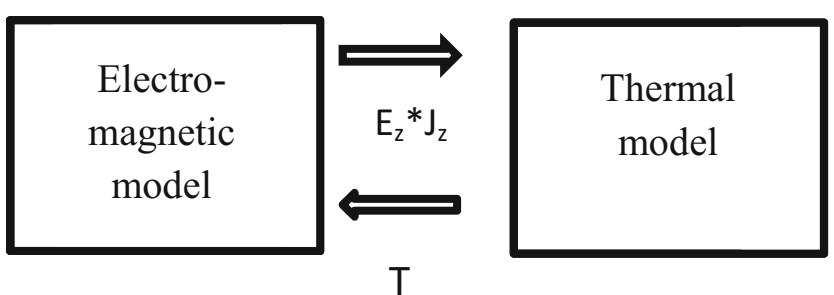

Fig. 6 Coupling between electromagnetic model and thermal model

where $h$ means convective heat transfer coefficient, which can be found in detail from [3]. $T_{\mathrm{S}}$ means the temperature of superconductor surface.

\subsubsection{Coupling of the Electromagnetic Model and Thermal Model}

In the simulation, the electromagnetic model and the thermal model are coupled together to calculate the characteristics of superconductors during quench. As shown in Fig. 6, the calculation results of electromagnetic model $E_{\mathrm{Z}}$ and $J_{\mathrm{Z}}$ are used as the input of thermal model. Also, the calculation results of thermal model $T$ is used as an input parameter of the electromagnetic model. Due to the powerful ability of COMSOL in solving the coupled problems, the two models can be coupled and calculated simultaneously in COMSOL, which can provide more precise results compared with the method based on a fast-forward scheme [6].

\section{Introduction of Fault Experiment and the Circuit Model}

\subsection{Introduction of Fault Experiment}

To test the current limiting performance of the SFCL prototype mentioned in Section 2, fault current tests are carried out using the experimental system shown in Fig. 7. The experimental system is consisted of a $3 \mathrm{MVA} / 15 \mathrm{kV} / 380 \mathrm{~V}$ transformer, a main switch, SFCL prototype, variable resistors R1 and R2, and a switch for short circuits, as shown in Fig. 7 [12]. The SFCL was tested under the voltage between phase and ground of the transformer, which is $220 \mathrm{~V} / 60 \mathrm{~Hz}$. In the fault current test, R1 and R2 can be adjusted to obtain suitable pre-fault currents and prospective fault currents, which vary from 0 to $10 \mathrm{kA}$. In the experiment, a steady current of 300 A flows in the circuit before the occurrence of short circuit, and the fault lasts for five cycles [12].
Table 3 Summary of applied fault currents

\begin{tabular}{llll}
\hline $\begin{array}{l}\text { Prospective } \\
\text { fault current/kA }\end{array}$ & $\begin{array}{l}\text { Limited } \\
\text { current/A }\end{array}$ & $\begin{array}{l}\text { SFCL } \\
\text { voltage/ }\end{array}$ & $\begin{array}{l}\text { Voltage per } \\
\text { rms }\end{array}$ \\
\hline 0.8 & 357.8 & 87 & 4.9 \\
1.0 & 390.3 & 113 & 5.9 \\
2.0 & 437.0 & 153 & 8.0 \\
3.4 & 448.3 & 176 & 9.9 \\
4.2 & 475.9 & 187 & 10.5 \\
5.7 & 475.9 & 194 & 11.0 \\
6.2 & 489.3 & 201 & 11.3 \\
7.0 & 492.1 & 203 & 11.5 \\
7.4 & 491.4 & 202 & 11.4 \\
\hline
\end{tabular}

In the fault experiment, the current limiting performance of the SFCL prototype is tested under different prospective fault currents from 0.8 to $7.4 \mathrm{kA}$. The test results are summarized in Table 3.

\subsection{Circuit Model}

To simulate the performance of SFCL in fault experiments as well as the corresponding response of the experimental circuit, an experimental circuit model is built and combined with the superconductor model mentioned in Section 2, as shown in Fig. 8 The experimental system shown in Fig. 7 is simulated with an electrical circuit built in electric circuit interface of AC/DC module of COMSOL, illustrated in Fig. 8a. Two variable resistors R1 and $\mathrm{R} 2$ are used to simulate the line resistor and load resistor separately.

As shown in Fig. 8b, the SFCL is expressed with a series connection of 16 elements in the electrical circuit, each one

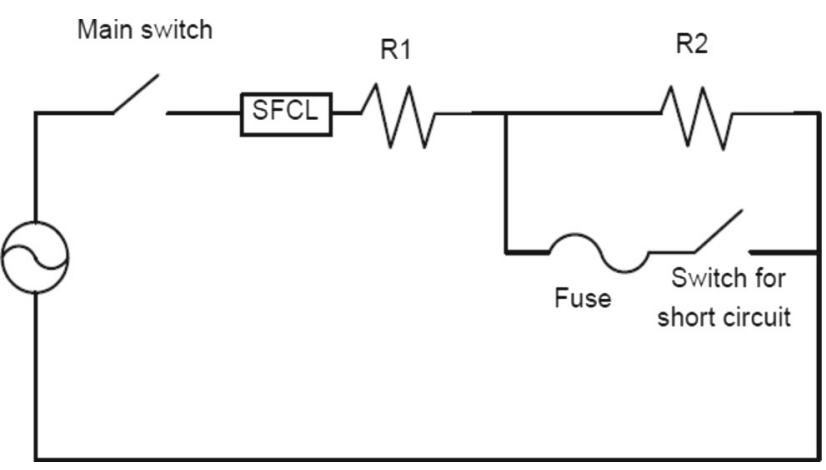

Fig. 7 Experimental system 


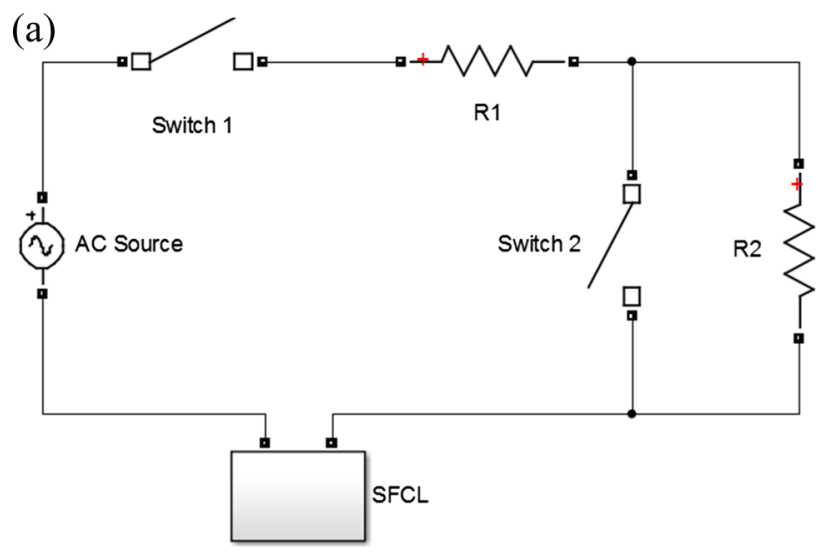

(b)

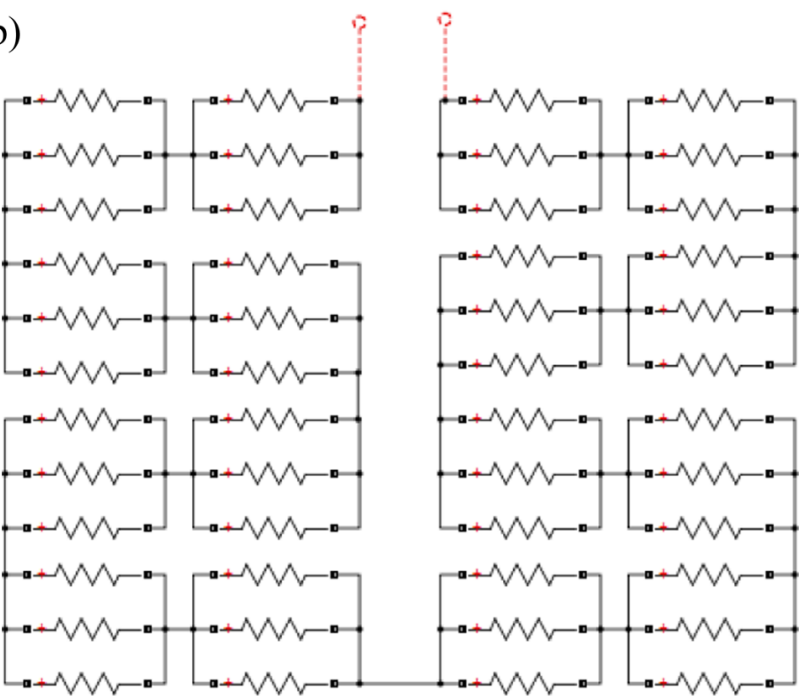

Fig. 8 Simulated electrical circuit: $\mathbf{a}$ is the schematic diagram of electrical circuit; $\mathbf{b}$ is the schematic diagram of the SFCL

consisted of three resistors, which represent YBCO layers, metal layers (metal layers mean a combination of the Ni5 at $\% \mathrm{~W}$ layer, $\mathrm{Ag}$ layer, mixture layer and SUS 316L layer), and shunt separately.

\subsection{Coupling of the Experimental Circuit Model and the Superconductor Model}

In the simulation, the superconductor model and the experimental circuit model are coupled together to simulate the current limiting performance of the SFCL prototype, as shown in Fig. 9. In each calculation step, the current flowing through the superconducting tapes of the SFCL prototype is transferred from the circuit model to the superconductor model to calculate the electrical and thermal characteristics of superconducting tapes. Also, the resistance of YBCO layers and the resistance of metal layers are transferred from the superconductor model to the experimental circuit model to continuously calculate the response of the experimental circuit.

The extraction of the current of the superconducting tapes from the experimental circuit model can be easily realized by summing up the current of the two resistors, which represent the YBCO layers and the metal layers in an element shown in Fig. 8b. The extractions of resistances of YBCO layers and the resistance of metal layers from the superconductor model are introduced below.

1. Extraction of the Resistance of Metal Layers The resistance of metal layers of a superconducting tape is regarded as the resistance of the six metal layers connected in parallel, which can be calculated with (14) and (15). As shown in (15), the resistivity of each metal layer is defined as functions of its average temperature.

$$
\begin{aligned}
& R_{\text {metal }}=\frac{1}{\sum_{i=1}^{i=6} \frac{1}{R_{i}(\bar{T})}} \\
& R_{i}(\bar{T})=\rho_{i}(\bar{T}) \times \frac{l}{S}
\end{aligned}
$$

\section{Extraction of Resistance of YBCO Layers}

The resistance of YBCO layers is calculated based on the assumption that the YBCO layers, and the metal layers are connected in parallel, meaning that the voltage drop on the two elements are the same. Therefore, the resistance of YBCO can be calculated by (16).

$R_{\mathrm{YBCO}}= \begin{cases}\min \left(\frac{R_{\text {metal }} \times I_{\text {metal }}}{I_{\mathrm{YBCO}}}, 29.544\right), & T_{\mathrm{YBCO}}<T_{\mathrm{c}} \\ 29.544 & , T_{\mathrm{YBCO}}>T_{\mathrm{c}}\end{cases}$

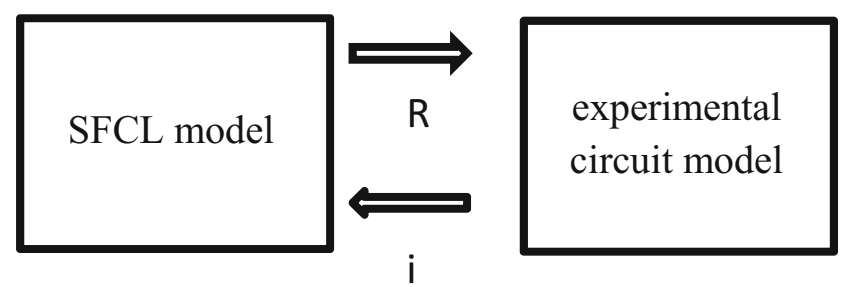

Fig. 9 Coupling of SFCL model and power system model 
where 29.544 is the normal resistance of the YBCO layers in room temperature, with unit ohm $\Omega$ When the temperature of YBCO is higher than the critical temperature, the superconductor completely loses its superconductivity, and thus the resistivity of YBCO increases to the normal resistivity. Although the normal resistivity of YBCO is temperature dependent, it is taken as $130 \mathrm{e}-8 \Omega \mathrm{m}$ here due to that the resistance of YBCO of each element is far larger than that of the metal layers and shunt after quench. When the temperature of YBCO is lower than the critical temperature, the resistance of YBCO depends on current density, temperature and magnetic field, with maximum value $29.544 \Omega$.

\section{Comparison Between Experimental Data and the Simulation Results}

In this work, different prospective fault current tests were simulated using the developed model. The details of the fault experiment with prospective current $7.4 \mathrm{kA}$, which contains the current limiting characteristics of SFCL prototype, the temperature trend, and the current distribution, are provided here. Then, to further validate the model, simulation results of SFCL at different prospective fault currents are summarized and compared with the experimental results.

\subsection{Simulation of the Fault Current Test With Prospective Fault Current 7.4 kA}

As shown in Figs. 10 and 11, the simulated SFCL current and SFCL voltage with prospective fault current $7.4 \mathrm{kA}$ are presented separately, which are compared with those in experiments. What is more, the simulation with Duron's equation is also presented to make a comparison. As shown in Fig. 10, the simulated SFCL currents with the new $E$ $J$ relationship are almost the same with that with Duron's equation. The two simulated currents are mostly consistent with the experiment. However, in the first half cycle of SFCL current, the simulated peak current is a bit smaller than that in experiment, which also occurs in the simulation of other fault current tests. This might be because the cooling effect of copper bus bar and the intermediate copper bars are not considered in the simulation. The cooling effect of copper bars can limit the temperature increase of superconducting layers to some extent, which again limits the increase of SFCL resistance, thus resulting in higher current peak.

In Fig. 11, the comparison between the simulated SFCL voltage and experimental SFCL voltage also indicated that

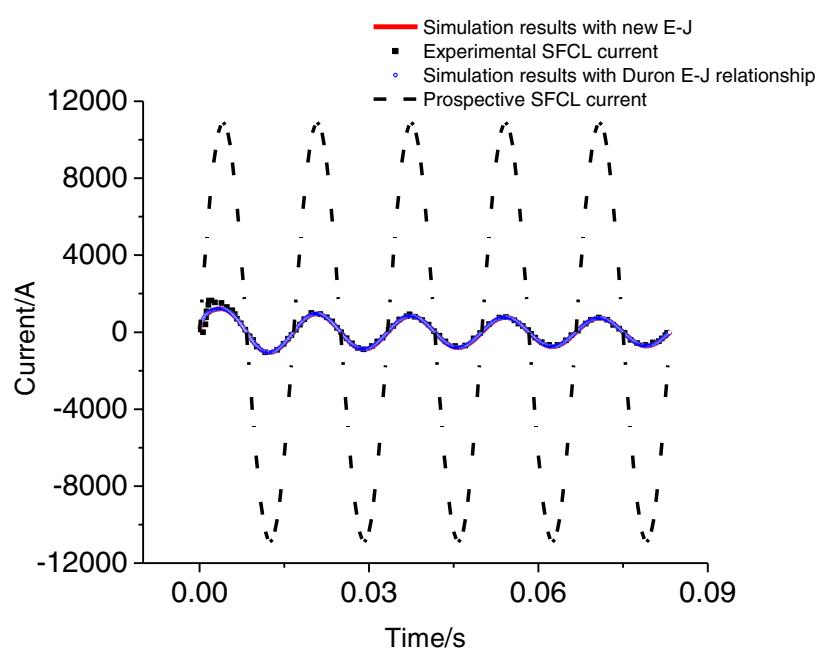

(a)

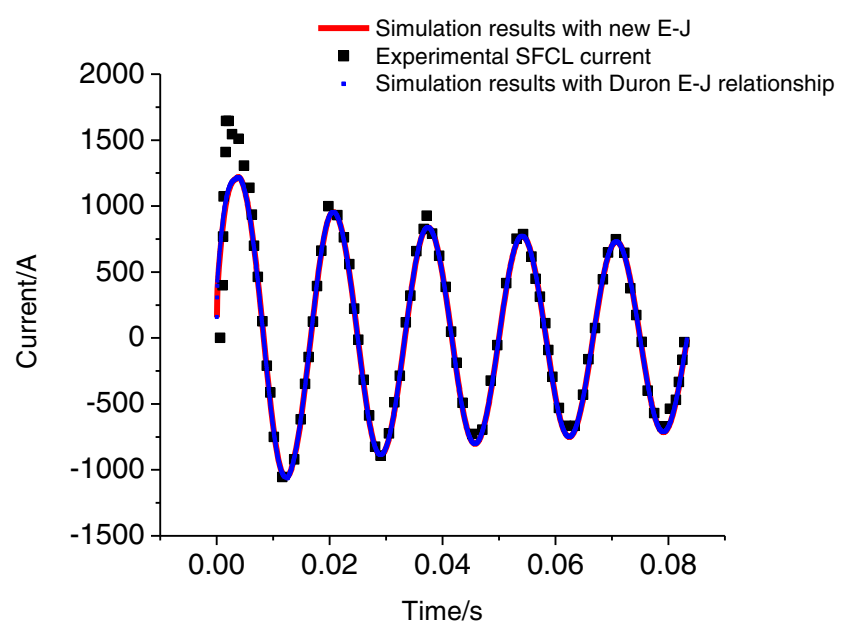

(b)

Fig. 10 Comparison between experimental and simulated SFCL current

the both the simulated SFCL voltages agree well with experiment.

Besides, the resistances of SFCL both in simulation and in experiment are also compared, as shown in Fig. 12. It clearly shows that the trends of both SFCL resistances are almost the same. Due to the fact that the temperature increases with fluctuation (see Fig. 13) and that the resistivity of $\mathrm{Ag}$, SUS 316L, and YBCO increase with the temperature, the resistance of SFCL also increases with fluctuations after quench.

As shown in Fig. 13, the average temperature of superconducting tapes increases with fluctuations in the test. This is mainly because the power supply is AC, which means the 


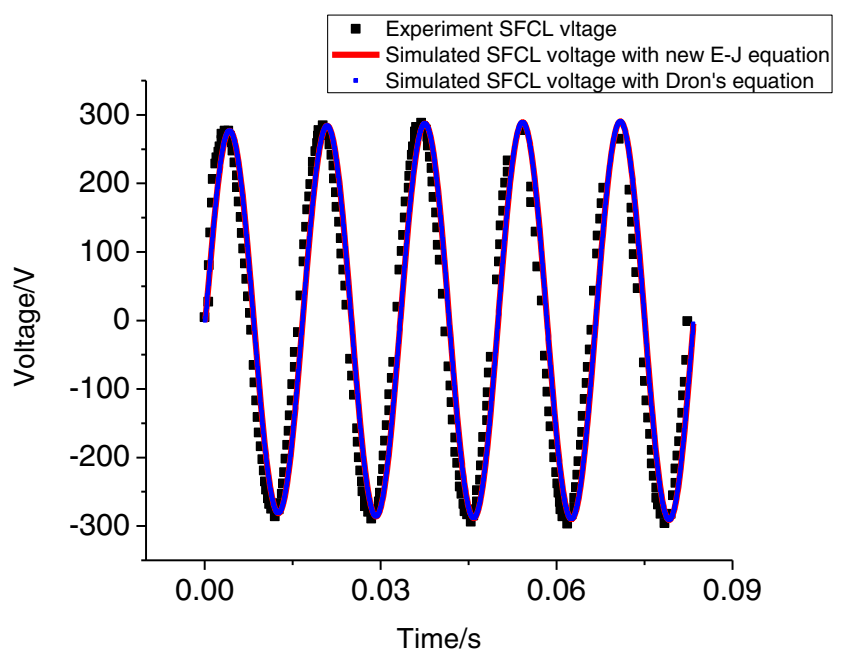

Fig. 11 Comparison of experimental and simulated SFCL voltage

heat dissipated in superconducting tape is proportional to $I^{2}$ (if the resistance of SC tape is regarded as a constant). Therefore, when current is crossing zero, the heat dissipated is small, which leads to small temperature increase of the superconductor. Moreover, when the current is at its peak value, whether it is positive or negative, the heat generated is very high, leading to a rapid temperature increase.

Also, the current distribution in different layers of tapes is provided in Fig. 14. It is clearly shown that current mainly flows through YBCO before quench and shunts to metal layer and the $180-\mathrm{m} \Omega$ shunt after quench of superconductor. What is more, due to the fact the metal layers resistance is far smaller than that of shunt, the current in metal layers is higher than that in the $180-\mathrm{m} \Omega$ shunt.

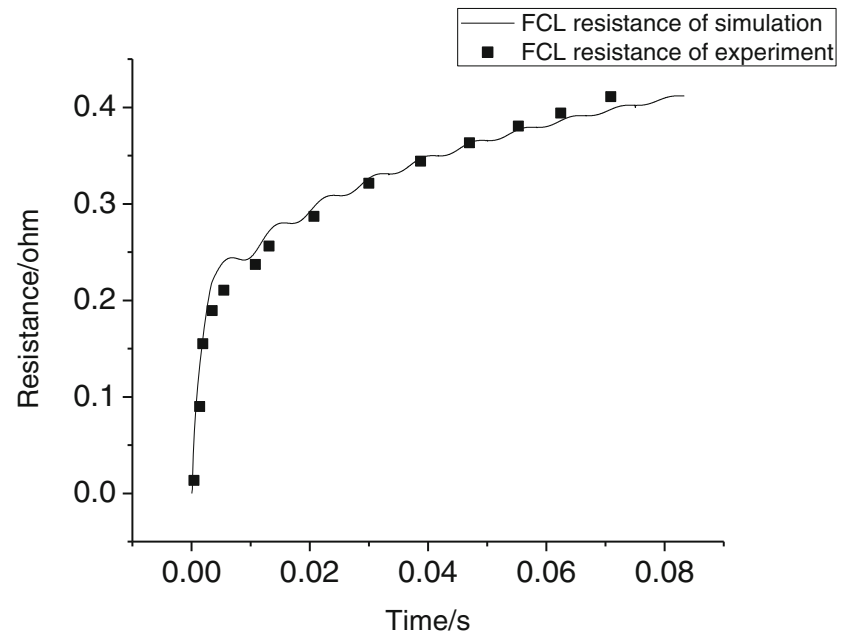

Fig. 12 Comparison between experimental and simulated SFCL resistance

\subsection{Simulation Result}

To further validate the simulation, the current limiting performance of the SFCL in different fault tests were simulated and compared with the experimental results shown in Table 3.

As shown in Fig. 15, the limited SFCL currents in simulation under different prospective fault currents were compared with that of experiments. The maximum calculation error is only $4.4 \%$ of the experimental results, which is very small when it is compared with the prospective current. The current limiting ratio, which is defined as the ratio of prospective current to limited current, was calculated and summarized in Fig. 16. It is clearly shown that the simulated current limiting ratios are almost the same as those in experiment. The SFCL voltages corresponding to the limited SFCL current in experiment and in simulation are also compared in Fig. 17, which suggests that the simulated SFCL voltage is almost the same as that in experiment. All these results show that the model performs well in simulating the performance of the SFCL.

\section{Discussion}

\subsection{Convergence Problem}

In the simulation, it is found that non-convergence sometimes occurs, especially when the temperature of superconductor approaches $90 \mathrm{~K}$ (critical temperature of superconductor). The reason causing this might be explained in the following way.

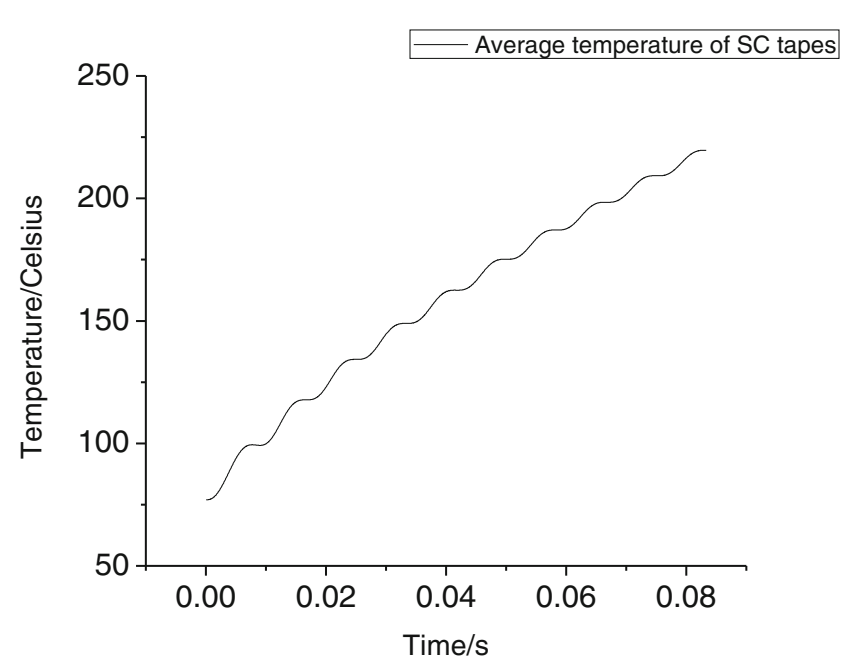

Fig. 13 Trend of average temperature during quench 


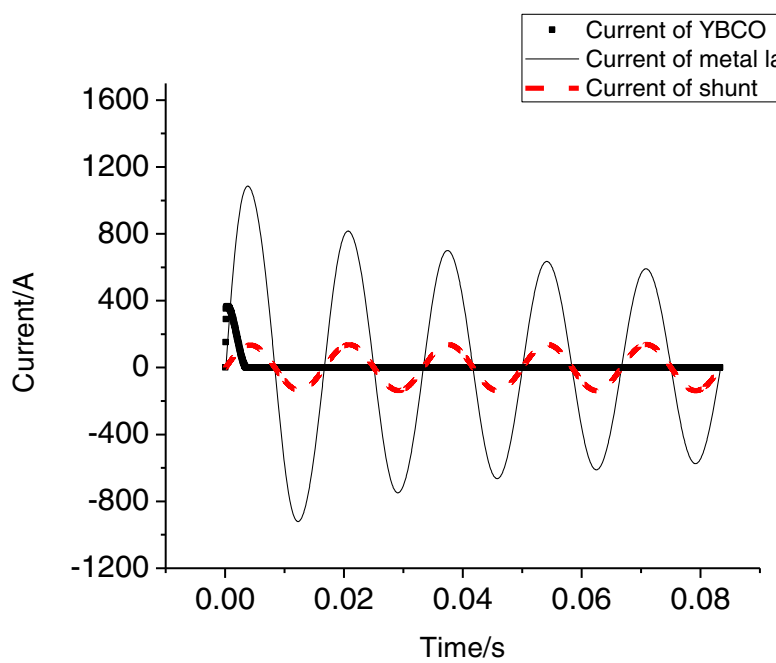

Fig. 14 Distribution of current in different layers

According to (8), the critical current density becomes very small as the temperature of superconductor approaches $90 \mathrm{~K}$. A small temperature vibration in local area that results from heat transfer in superconductor might lead to a great change of local resistivity, which then leads to the change of current density and magnetic field distribution in local area, making the calculation very difficult to converge.

To solve the problem, it is necessary to decrease the slope of the $E$ - $J$ curve of superconductor when the current density of superconductor is higher than critical current

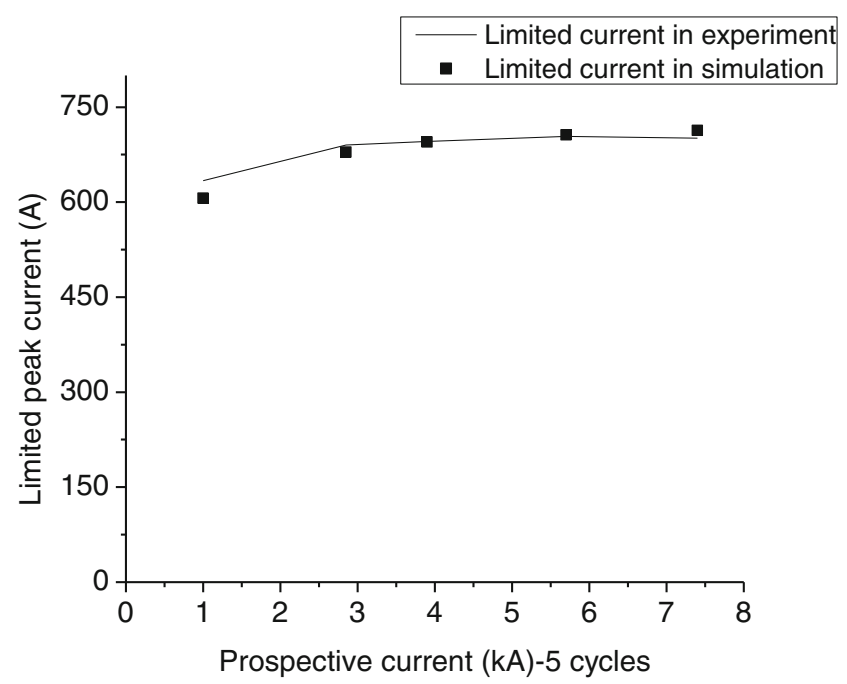

Fig. 15 Comparison of limited peak current in experiment and in simulation

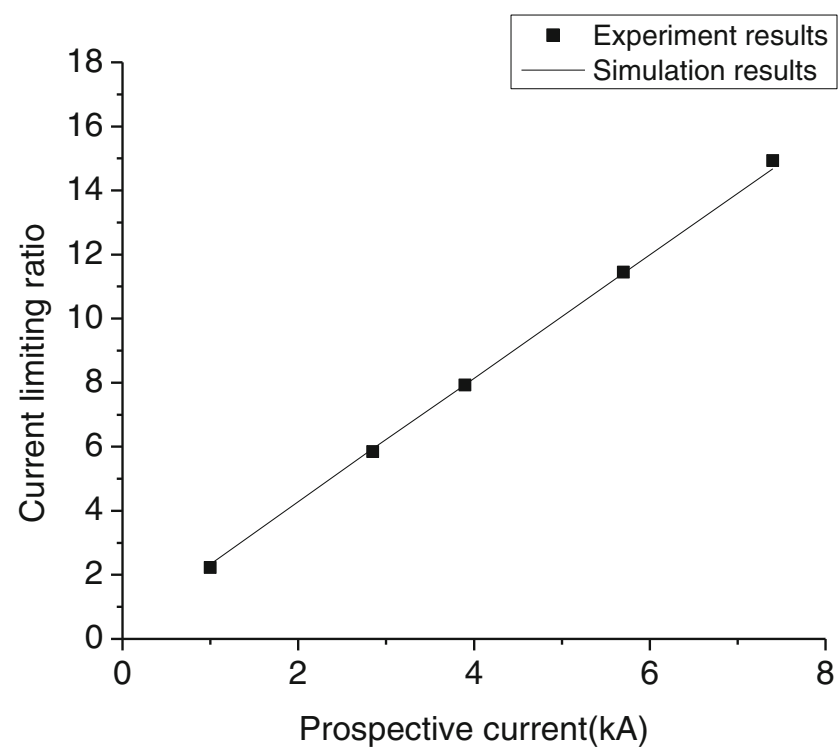

Fig. 16 Comparison of current limiting ratio in experiment and in simulation

density while keep the basic characteristics of superconductor: negligible electrical field when the current is smaller than the critical current density. The decrease of the slope of $E-J$ curve when $J>J_{\mathrm{c}}$ can effectively limit the resistivity change of local superconductor resulting from the temperature vibration, thus enhance the convergence. Therefore, in this paper, a new $E-J$ curve with smaller slope is introduced.

Another method that can enhance the convergence of calculation is that the temperature rise rate of each element

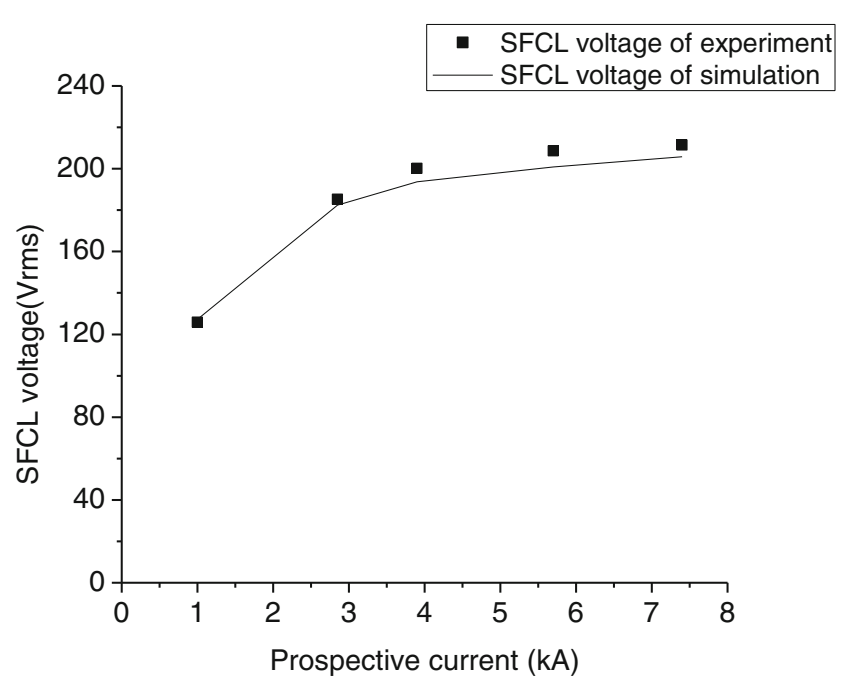

Fig. 17 Comparison of SFCL voltage in experiment and simulation 
should be decreased during calculation. This can be realized by increasing the mesh density of the superconducting tape, especially in the thickness direction. It is because, according to the simulations, the temperature gradient in the thickness direction is far larger than that in the width direction.

\subsection{Computation Time}

The calculations in this paper are sometimes timeconsuming, mainly because that the real geometry of superconducting tape is used and all the PDEs of the superconductor model and the equations of the experimental circuit model are calculated simultaneously. Generally speaking, it usually takes about $40-70 \mathrm{~h}$ to calculate five cycles with computer with Intel Core i5 3.4-GHz processor and 8-GB memory.

The computation time depends on many factors, such as the prospective fault current, mesh number, and the time steps. Sometimes, a small change in the mesh, aiming to enhance the convergence in the calculation, might result in a significant increase of computation time. Therefore, the increase of the calculation speed is an important task in future research.

\section{Conclusion}

In this paper, a $220-\mathrm{V}$ resistive SFCL prototype is built and tested under different fault currents. To simulate the macroscopic characteristics of the SFCL prototype, a finite element model coupling a superconductor model and an experimental circuit model is proposed. In the simulation, a new $E-J$ relationship is provided to enhance the convergence of calculation.

The simulation realizes the real-time data exchange between the coupled models during calculations, thus leading to more precise calculation results. The simulated results of different fault current tests are compared with those in experiments, which show that the provided model performs well in simulating the current limiting performance of the SFCL prototype. What is more, the model proposed can also be extended to simulating the performance of SFCL in real power grids, considering that the simulated experimental circuit can be regarded as a simple power grid.

However, the calculation is sometimes timeconsuming, which is mainly because the real geometry of superconducting tapes are used and all the coupled models are calculated simultaneously.

In the next step, the simulation of superconducting tape will be extended from 2D to 3D, which makes it possible to take into account more factors, such as the influence of copper bars and to study the quench process of superconductor in more details.

\section{Appendix}

\section{Detailed Temperature-dependent Variables are Provided Here}

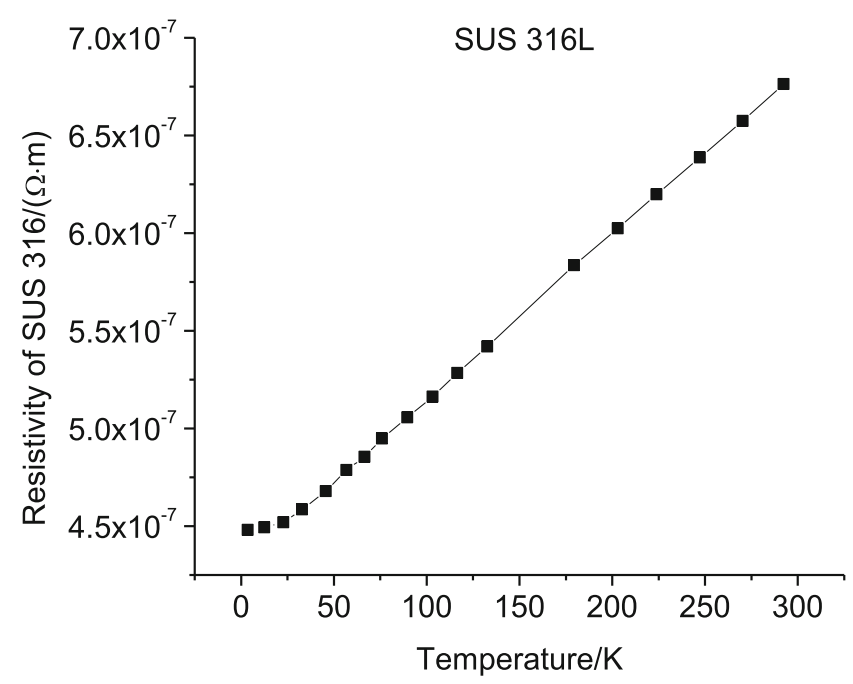

Fig. 18 Resistivity of SUS 316L [14]

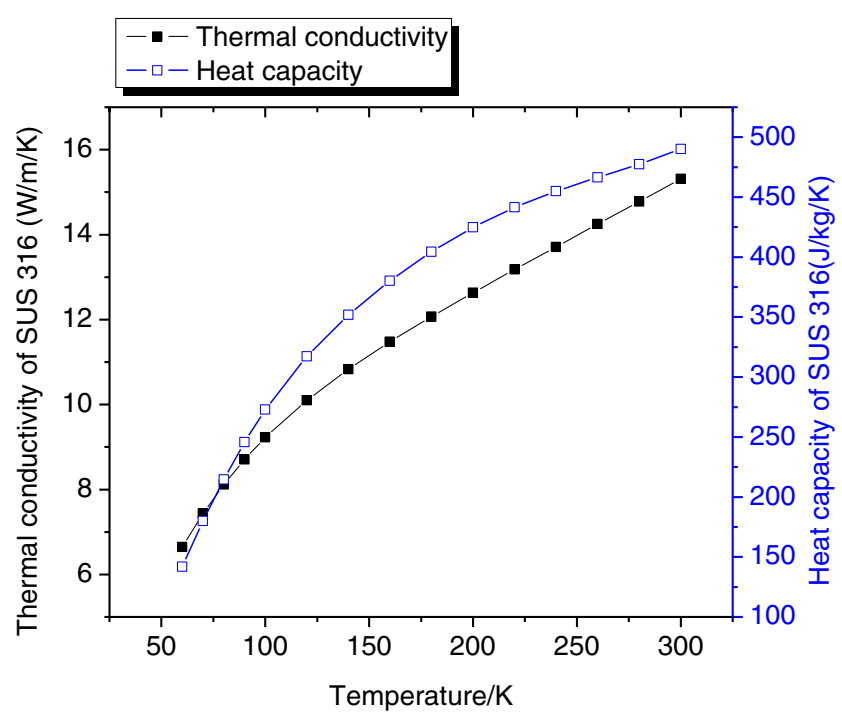

Fig. 19 Thermal conductivity and heat capacity of SUS $316[15,16]$ 


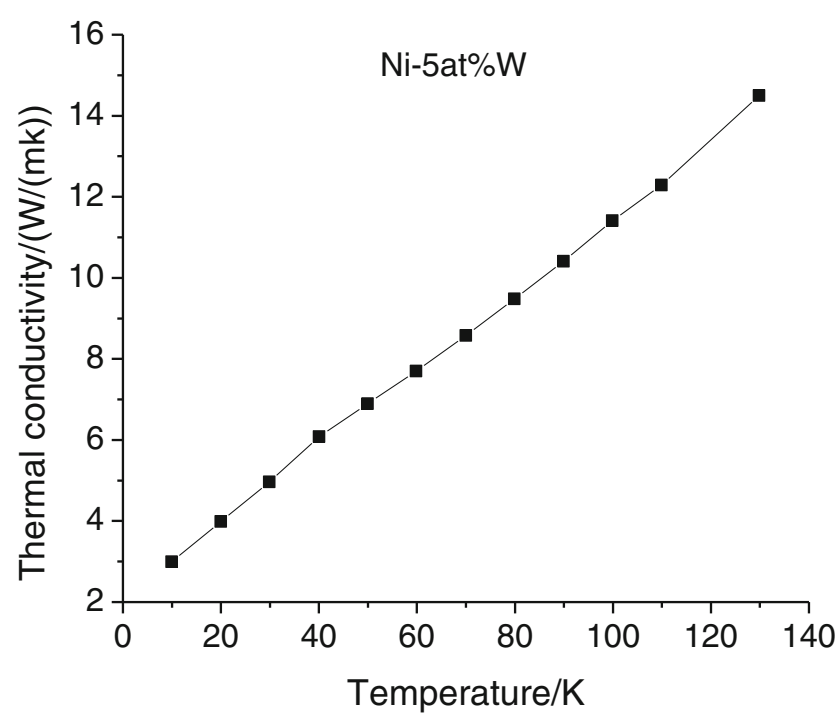

Fig. 20 Thermal conductivity of Ni-5at \%W [21]

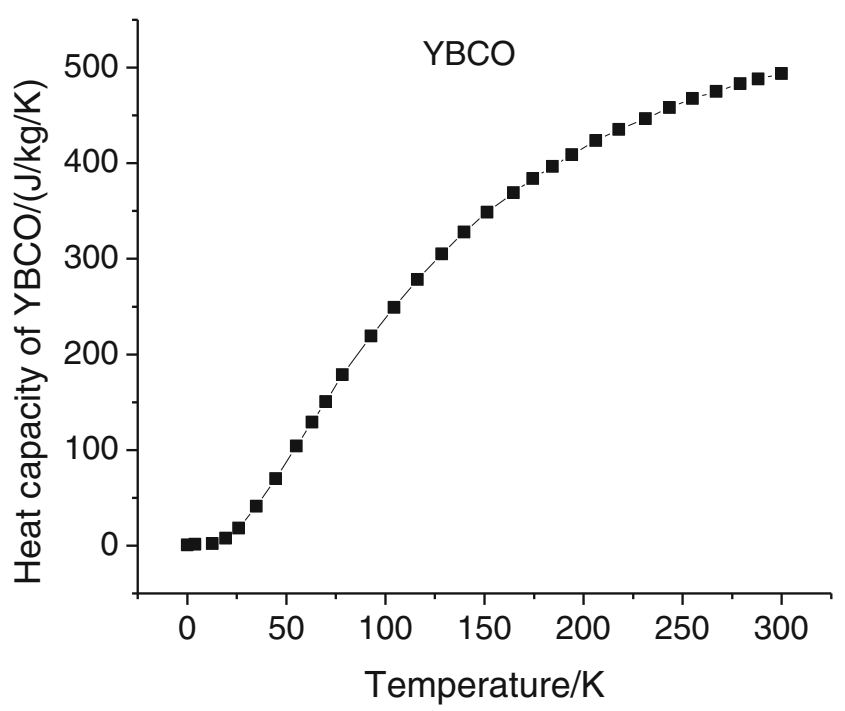

Fig. 21 Heat capascity of YBCO [22]

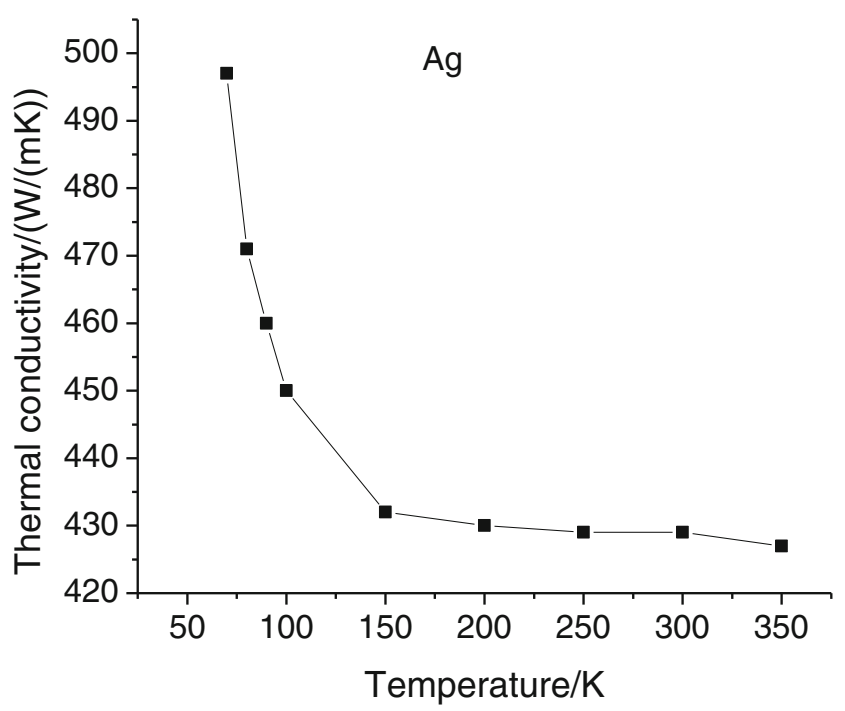

Fig. 22 Thermal conductivity of silver [24, 25]

\section{References}

1. Kovalsky, L., Yuan, X., Tekletsadik, K., Keri, A., Bock, J., Breuer, F.: Applications of superconducting fault current limiters in electric power transmission systems. IEEE Trans. Appl. Supercond. 15, 2130-2133 (2005)

2. Roy, F., Pérez, S., Therasse, M., Dutoit, B., Sirois, F., Decroux, M., et al.: Quench propagation in coated conductors for fault current limiters. Phys. C: Supercond. 469, 1462-1466 (2009)

3. Roy, F., Dutoit, B., Grilli, F., Sirois, F.: Magneto-thermal modeling of second-generation HTS for resistive fault current limiter design purposes. IEEE Trans. Appl. Supercond. 18, 29-35 (2008)

4. Zhang, M., Matsuda, K., Coombs, T.: New application of temperature-dependent modelling of high temperature superconductors: Quench propagation and pulse magnetization. J. Appl. Phys. 112, 043912 (2012)

5. Zhang, M., Kim, J.-H., Pamidi, S., Chudy, M., Yuan, W., Coombs, T.: Study of second generation, high-temperature superconducting coils: Determination of critical current. J. Appl. Phys. 111, 083902 (2012)

6. Duron, J., Grilli, F., Antognazza, L., Decroux, M., Dutoit, B., Fischer, Ø.: Finite-element modelling of YBCO fault current limiter with temperature dependent parameters. Supercond. Sci. Technol. 20, 338 (2007)

7. Chang, K.S., Park, D.K., Yang, S.E., Kim, Y.J., Chu, S.Y., Ahn, M.C., et al.: Characteristic comparison of the superconducting fault current limiter with various bypass reactors. IEEE Trans. Appl. Supercond. 20, 1190-1193 (2010)

8. Chan, W.K., Masson, P.J., Luongo, C., Schwartz, J.: Threedimensional micrometer-scale modeling of quenching in highaspect-ratio coated conductor tapes-Part I: model development and validation. IEEE Trans. Appl. Supercond. 20, 2370-2380 (2010)

9. Chan, W.K., Schwartz, J.: Three-dimensional micrometer-scale modeling of quenching in high-aspect-ratio coated conductor tapes-Part II: influence of geometric and material properties and implications for conductor engineering and magnet design. IEEE Trans. Appl. Supercond. 21, 3628-3634 (2011)

10. Badel, A., Antognazza, L., Therasse, M., Abplanalp, M., Schacherer, C., Decroux, M.: Hybrid model of quench propagation in coated conductors for fault current limiters. Supercond. Sci. Technol. 25, 095015 (2012)

11. Antono Morandi, M.F., Gholizad, B.abak.: Modeling of the resistive type superconducting fault curent limiter for power system analysis and optimization, in HTS Modelling 2014 (2014)

12. Baldan, C.A., Lamas, J.S., Shigue, C.Y.: Test of a modular fault current limiter for $220 \mathrm{~V}$ line using YBCO coated conductor tapes with shunt protection. IEEE Trans. Appl. Supercond. 21, 12421245 (2011)

13. Nguyen, N., Tixador, P.: A YBCO-coated conductor for a fault current limiter: architecture influences and optical study. Supercond. Sci. Technol. 23, 025008 (2010)

14. Umezawa, O., Ishikawa, K.: Electrical and thermal conductivities and magnetization of some austenitic steels, titanium and titanium alloys at cryogenic temperatures. Cryogenics 32, 873-880 (1992)

15. Wang, X., Trociewitz, U., Schwartz, J.: Self-field quench behaviour of $\mathrm{YBa} 2 \mathrm{Cu} 3 \mathrm{O} 7-\delta$ coated conductors with different stabilizers. Supercond. Sci. Technol. 22, 085005 (2009)

16. (2014-2015). Handbook of chemistry and physics. Available: http://www.hbcpnetbase.com/

17. Sn 62 Sn-Pb-Ag Solder Alloy. Available: http://www.com/search/ datasheet.aspx ?matguid=703a13aad9d444bc988c4d6f96675045\& $\mathrm{ckck}=1$ 
18. Sn $63 \mathrm{Sn}-\mathrm{Pb}$ solder alloy. Available: http://www.matweb.com/ search/datasheet.aspx?MatGUID=c1d1b91a360748bf9944a0c771 e8d5b2

19. E. J. W.: Experimental techniquies for low-temperature measurements. Oxford University Press, Oxford (2006)

20. Park, D.K., Bang, J.S., Yang, S.E., Ko, T.K., Yoon, Y.S., Ahn, M.C., et al.: Theoretical and experimental analysis of AC loss characteristic of bifilar pancake coil with coated conductor. IEEE Trans. Appl. Supercond. 18, 1232-1235 (2008)

21. Hoffmann, C., Strickland, N., Pooke, D., Gannon, J., Carter, B., Otto, A.: Thermal conductivity of $2 \mathrm{G}$ HTS wires for current lead applications. In: Journal of Physics: Conference Series, p. 022015 (2010)

22. Junod, A., Graf, T., Sanchez, D., Triscone, G., Muller, J.: Specific heat of the superconductor Yba2Cu4O8 from 1.5 to 300K. Phys. B: Cond. Matter 165-166, 1335-1336, 8// (1990). Part 2
23. Electrical resistivity and conductivity. Available: http://en. wikipedia.org/wiki/Electrical_resistivity_and_conductivity

24. Griffiths, D.J.R.: Introduction to electrodynamics vol. 3: Prentice Hall Upper Saddle River, NJ (1999)

25. Serway, R.A.: Principles of physics. Fort Worth, Texas; London: Saunders College Pub, ISBN 0-03-020457-71998

26. Hong, Z., Campbell, A., Coombs, T.: Numerical solution of critical state in superconductivity by finite element software. Supercond. Sci. Technol. 19, 1246 (2006)

27. Duron, J., Grilli, F., Dutoit, B., Stavrev, S.: Modelling the $E-J$ relation of high- $T_{\mathrm{c}}$ superconductors in an arbitrary current range. Phys. C: Supercond. 401, 231-235 (2004)

28. Paul, W., Chen, M., Lakner, M., Rhyner, J., Braun, D., Lanz, W., et al.: Superconducting fault current limiter: applications, technical and economical benefits, simulations and test results, CIGRÉ session 2000 (2000) 